from an EOF analysis of SST anomalies in the tropical Pacific, which yields two dominant principal-component time series and two associated anomaly patterns ${ }^{5}$. They then use a linear combination of these principle components and patterns to identify an individual EP-El Niño centre for each climate model. Finally, they introduce an EP-El Niño index for each model, which represents the model's EP-El Niño centre and pattern. The authors report that a reasonable consensus emerges: 24 of the 34 available models (71\%) predict an increase in EP-El Niño SST variability under a climate-change scenario (known as RCP8.5) that assumes greenhouse-gas emissions will continue to rise steeply throughout the twentyfirst century.

However, most of the models underestimate the SST skewness. Cai et al. show that nonlinear processes responsible for the negative skewness in the central Pacific are tightly connected to those for the positive skewness in the eastern Pacific, and are represented by a nonlinear relationship between the two principle components. Focusing on 17 models that simulate these nonlinear processes realistically, the authors find an even stronger consensus: 15 of the 17 models ( $88 \%)$ predict a rise in EP-El Niño SST variability under the RCP8.5 emissions scenario.

Cai and colleagues' work shows that, under global-warming conditions, warming occurs more quickly at the surface layer of the ocean than in subsurface layers ${ }^{6}$. This increases the vertical temperature gradient of the ocean, which in turn enhances the dynamical coupling between the atmosphere and the ocean. Consequently, the equatorial ocean-atmosphere system becomes more efficient at converting stochastic fluctuations in winds into a potential EP-El Niño event, leading to increased EP-El Niño variability. The authors' results also indicate that, by a similar mechanism, SST variability in the central Pacific is enhanced (albeit not as strongly as in the eastern Pacific). This translates into an increased frequency of CP-El Niño events and of extreme La Niña events - a conclusion that is consistent with previous studies ${ }^{7,8}$.

The authors' finding of increased EP-El Niño variability under global warming represents a milestone in climate research, and will inspire studies of the worldwide impact of future changes in El Niño events. However, the work also raises many questions. For example, why do so many climate models fail to simulate the nonlinear processes associated with the SST skewness? What leads to the large discrepancies in the model simulations? And how sensitive is the reported consensus to future models? Cai and colleagues' results therefore need to be assessed further as other model simulations become available. Nevertheless, the projection of more-frequent and stronger El Niño events must be taken seriously, as we prepare to deal with the consequences of global warming. -
Yoo-Geun Ham is in the Department of Oceanography, Chonnam National University, Gwangju, South Korea.

e-mail:ygham@jnu.ac.kr

1. Collins, M. et al. Nature Geosci. 3, 391-397 (2010).

2. Cai, W. et al. Nature Clim. Change 5, 849-859 (2015).

3. Cai, W. et al. Nature $564,201-206$ (2018).
4. Ham, Y.-G. \& Kug, J.-S. Clim. Dyn. 39, 383-398 (2012)

5. Takahashi, K., Montecinos, A., Goubanova, K. \& Dewitte, B. Geophys. Res. Lett. 38, L10704 (2011).

6. An, S.-I., Kug, J.-S., Ham, Y.-G. \& Kang, I.-S. J. Clim. 21, 3-21 (2008).

7. Cai, W. et al. Nature Clim. Change 5, 132-137 (2015).

8. Yeh, S.-W. et al. Nature 461, 511-514 (2009).

9. Rayner, N. A. et al. J. Geophys. Res. 108, 4407 (2003).

\title{
VIROLOGY
}

\section{How cells hush a viral invader}

Viruses can insert a copy of their genetic sequence into a host cell's genome. If the insertion fails, gene expression of unintegrated viral DNA in the nucleus is silenced. How this process occurs has now been uncovered. SEE LETTER P.278

\section{PARINAZ FOZOUNI \& MELANIE OTT}

$\mathrm{V}$ iruses known as retroviruses encode their genetic blueprint in the form of RNA. When these viruses enter a host cell, a viral enzyme generates a DNA version of this RNA sequence that can be permanently integrated into the host-cell genome, despite the host cell's efforts to avoid this outcome. Gene silencing provides a vigorous form of host defence against viral DNA that reaches the nucleus but does not successfully integrate into the genome; however, this mechanism is poorly understood. Such gene silencing can limit gene-therapy approaches that use engineered unintegrated retroviruses. Zhu et al. ${ }^{1}$ reveal on page 278 that the evolutionarily conserved DNA-binding protein NP220 has a central role in silencing the transcription of unintegrated retroviruses.

When unintegrated retroviral DNA enters the host-cell nucleus it rapidly binds to histone proteins ${ }^{2}$, which package DNA, suggesting that retroviral sequences are subject to regulation even before any attempted integration occurs. To identify host factors that might mediate the silencing of unintegrated retroviral DNA, Zhu et al. used a gene-editing technique called CRISPR-Cas9. This enabled the authors to eliminate expression of individual genes across the entire genome of human cells grown in vitro and to test the effect of this on the silencing of viral genes.

The experimental results led the authors to focus on five host proteins. One was the DNAbinding protein ${ }^{3}$ NP220, which is found in the nucleus. Three other proteins of interest MPP8, TASOR and PPHLN1 - form a multiprotein structure called the HUSH complex that has been previously associated ${ }^{4}$ with maintaining the dormant state of integrated HIV, and in contributing to the silencing of integrated

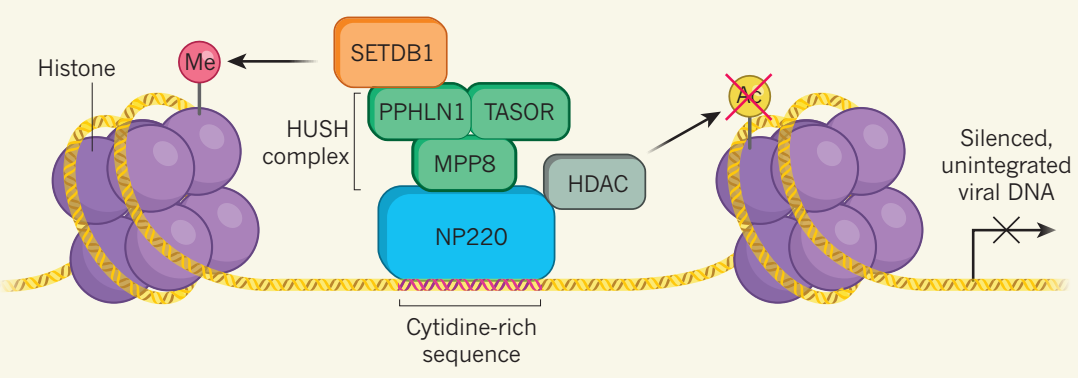

Figure 1 | A protein complex that suppresses viral-gene expression. Zhu et al. investigated how human cells silence gene expression of viral DNA that is not integrated into the host-cell genome. The authors report that viral DNA regions that are rich in the DNA building block cytidine are bound by the DNAbinding protein NP220. This protein is associated with the proteins PPHLN1, TASOR and MPP8 that form the HUSH complex (green). Another protein associated with this group is the enzyme SETDB1, which can add methyl groups (Me) to histone proteins that package viral DNA. This type of histone modification usually helps to silence gene expression. The removal of acetyl groups (Ac) on histones also represses gene expression. The authors report that histone deacetylase (HDAC) enzymes that catalyse the removal of such acetyl groups are found in association with NP220. 
retroviral sequences called retroelements ${ }^{5,6}$. The fifth protein was SETDB1, a methyltransferase enzyme that can modify histone proteins and interact with the HUSH complex to block the transcription of newly integrated retroviral $\mathrm{DNA}^{4-6}$.

The authors tested the function of each of these proteins in human cells grown in vitro that were infected with murine leukaemia virus, a model retrovirus that was used in early attempts at retroviral gene therapy ${ }^{7}$. They found that NP220 binds unintegrated retroviral DNA (Fig. 1) and, on binding, recruits the HUSH complex. This complex then recruits SETDB1 to deposit methyl modifications on histone proteins bound to viral DNA. These histone modifications are associated with the suppression of gene expression. Zhu and colleagues also discovered that NP220 recruits two enzymes, called HDAC1 and HDAC4, from a family of enzymes called histone deacetylases, which catalyse the removal of acetyl groups from histones. A decrease in the level of histone acetylation can repress gene expression.

The authors tested the effect of depleting either NP220 or HDAC1 and HDAC4 in human host cells infected with different types of retrovirus. For example, they tested HIV, which is from a different genus of retroviruses from that of murine leukaemia virus, and found that the depletion of these components caused an increase in gene expression from the unintegrated retrovirus. However, the depletion of HUSH-complex components or SETDB1 did not cause increased expression of the unintegrated viral sequence in this scenario. A similar pattern of results was obtained when the authors conducted the same type of study using Mason-Pfizer monkey virus, which belongs to yet another genus of retroviruses. This suggests that the role of NP220 is evolutionarily conserved, but that the proteins that might act with NP220 to silence viral gene expression can vary depending on the retrovirus.

NP220 can bind sequences in doublestranded DNA that are rich in the nucleoside cytidine ${ }^{3}$. Zhu and colleagues report that there are sequences rich in the DNA building block cytidine in repeat sequences called long terminal repeats (LTRs) at the ends of murine leukaemia virus, HIV and Mason-Pfizer monkey virus sequences, and that these cytidine-rich LTR sequences can serve as binding sites for NP220. When the authors tested the effect of depleting NP220 in human cells infected with Rous sarcoma virus (from another retrovirus genus), which has cytidine-poor LTRs, this depletion did not affect the gene expression of unintegrated viral DNA. This suggests that NP220 needs cytidine-rich DNA sequences to bind and silence viral genes. However, the NP220-independent silencing of gene expression of unintegrated Rous sarcoma viral DNA was found to be SETDB1 dependent because the deletion of the gene encoding SETDB1 led to increased gene expression of the viral DNA.
It would be interesting to learn more about how unintegrated Rous sarcoma virus is silenced through this NP220-independent mechanism. Together, these studies reveal that NP220 or its interacting partners can silence unintegrated DNA from a range of retroviruses.

The HUSH proteins and SETDB1 are involved $^{4-6}$ in the transcriptional silencing of integrated retroviral elements. Whether NP220 also acts to silence the expression of integrated retroviral DNA remains to be determined. This is a possibility, given that the cytidine-rich binding motifs for NP220 are preserved in viral LTRs after their integration into a host genome. Clearly, there is more to investigate about the silencing mechanism uncovered by Zhu and colleagues.

The high level of evolutionary conservation of NP220 proteins across vertebrate species is noteworthy. The origin of retroviruses is at least as ancient as that of vertebrates ${ }^{8}$. Perhaps NP220 represents an ancestral defence system to tackle invading retroviruses. But, in turn, some retroviruses could have evolved mechanisms to evade such gene silencing, which might explain why Rous sarcoma virus has lost cytidine-rich LTRs that would provide NP220 binding sites. Zhu and colleagues' study might inspire new ways to prevent the silencing of foreign DNA for use in gene-therapy applications. Importantly, it also provides clues to the evolutionary arms race between humans and retroviruses, and offers insights into a mechanism that might offer protection against disease-causing viruses.

Parinaz Fozouni and Melanie Ott are at the Gladstone Institutes, San Francisco, and in the Department of Medicine, University of California, San Francisco, California 94143, USA.

e-mail:melanie.ott@gladstone.ucsf.edu

1. Zhu, Y., Wang, G. Z., Cingöz, O. \& Goff, S. P. Nature 564, 278-282 (2018).

2. Wang, G. Z., Wang, Y. \& Goff, S. P. Cell Host Microbe 20, 798-809 (2016).

3. Inagaki, H. et al. J. Biol. Chem. 271, 12525-12531 (1996).

4. Tchasovnikarova, I. A. et al. Science 348, 1481-1485 (2015).

5. Robbez-Masson, L. et al. Genome Res. 28, 836-845 (2018).

6. Liu, N. et al. Nature 553, 228-232 (2018).

7. Thomas C. E., Ehrhardt, A., \& Kay, M. A. Nature Rev. Genet. 4, 346-358 (2003)

8. Aiewsakun, P. \& Katzourakis, A. Nature Commun. 8, 13954 (2017).

This article was published online on 28 November 2018.

\section{Adrenaline fuels a cytokine storm}

Attempts to boost the body's antitumour immune responses can trigger a harmful inflammatory reaction called a cytokine storm. New insights into the mechanisms involved might help to prevent this problem. SEE LETTER P.273

\section{STANLEY R. RIDDELL}

$\mathrm{M}$ any newly developed, potent cancer therapies aim to harness an immune response to target tumours ${ }^{1}$. However, a common problem with such immunotherapy approaches is the development of a severe inflammatory response called a cytokine storm ${ }^{2,3}$, in which levels of proteins called cytokines become abnormally high. This results in fever, low blood pressure, heart problems and, in some cases, organ failure and death. There is therefore great interest in understanding the underlying mechanisms to develop ways of preventing cytokine storms without altering the effectiveness of anticancer treatments. On page 273, Staedtke et al. ${ }^{4}$ reveal that the protein ANP can block cytokine storms, and they uncover a self-amplifying production loop in immune cells that generates a class of molecule called catecholamines, which includes the hormone adrenaline (also known as epinephrine). They report that this catecholamine production helps to initiate and maintain cytokine storms.

When immune cells recognize a molecule that indicates a possible threat, they release cytokines that promote inflammation and orchestrate host defence ${ }^{5}$. One antitumour treatment that can trigger a cytokine storm uses a bacterium called Clostridium novyi-NT, which tracks to the low-oxygen environments found in certain tumours and releases spores that cause tumour-cell death ${ }^{6}$. Determining the correct bacterial dosage is difficult, and mice that have large tumours and receive a high dose of C. novyi-NT often develop a fatal cytokine storm that cannot be prevented by using inhibitor molecules to block the actions of cytokines or their receptors ${ }^{6}$.

To determine whether some known antiinflammatory proteins could block a cytokine storm, Staedtke and colleagues engineered C. novyi-NT to secrete anti-inflammatory proteins and tested whether any of these bacteria could treat tumours effectively without 